\section{Wirkung wiederholter Leberresektionen auf die Diäthylnitrosamin-induzierte Carcinogenese}

\author{
H. Rabes, P. Scholze und R. Hartenstein \\ Pathologisches Institut der Universität München
}

(Z. Naturforsch. 25 b, 896 [1970] ; eingegangen am 29. April 1970)

Es wird angenommen, daß ein kontinuierlicher pathologischer Proliferationsreiz die Entstehung von Tumoren begünstigen kann. Wiederholt ist experimentell untersucht worden, ob eine Wachstumsstimulierung während oder nach Einwirkung chemischer Carcinogene zu vermehrtem Auftreten von Tumoren und $\mathrm{Ab}$ kürzung der Überlebenszeit der Versuchstiere führt. Die Ergebnisse sind uneinheitlich ${ }^{1-4}$. Offenbar ist der Zeitpunkt der Wachstumsanregung von Bedeutung.

Nach Fütterung des Carcinogens Diäthylnitrosamin ${ }^{5}$ werden etwa um den 40. Tag Glucose-6-Phosphatasedefiziente Hepatocyten in umschriebenen Bezirken der Leber sichtbar ${ }^{6,7}$. Sie werden als direkte Vorläufer hepatocellulärer Carcinome gedeutet ${ }^{8,9}$. Die Zellen der Enzymausfallsbezirke zeigen nach einer partiellen Hepatektomie ${ }^{10}$ der Versuchstiere einen signifikanten Anstieg der Proliferation ${ }^{11}$. Mehrfach aufeinanderfolgende partielle Hepatektomien in noch früheren Perioden der Carcinogenese vor dem Auftreten von Glucose-6-Phosphatase-defizienten Zellen hatten dagegen eine andere Wirkung.

44 Ratten (männlich, Wistar AF/Han, $150-170 \mathrm{~g}$ ) erhielten bei Altromin-Standarddiät $5 \mathrm{mg}$ Diäthylnitrosamin (D DÄNA erfolgte bis zum Tod der Tiere mit jeweils 3-tägigen Unterbrechungen am 8., 15., 22., 29. und 36. Tag. Je 7-8 Ratten wurden zu einer Gruppe zusammengefaßt. Außer den Kontrollen wurden die Ratten aller Gruppen am 8. Tag partiell leberreseziert. Bei den folgenden partiellen Hepatektomien am 15., 22., 29. und 36. Tag wurde jeweils eine weitere Gruppe nicht mehr operiert, so daß außer den Kontrolltieren alle Ratten 1- bis 5-mal zwischen 8. und 36. Tag nach Fütterungsbeginn operiert waren. Die Menge des resezierten Lebergewebes betrug in Feuchtgewichtsprozenten der Gesamtleber vor der jeweiligen Operation $14,7 \% \pm 1,7 \quad$ (Standardabweichung) $; \quad 17,9 \% \pm 4,3 ;$ $32,2 \% \pm 6,5 ; 17,8 \% \pm 4,1 ; 41,8 \% \pm 14,1$.

1 A. D. Glinos, N. L. R. Bucher, and J. C. Aub, J. exp. Medicine 93, 313 [1951].

2 J. O. Laws, Brit. J. Cancer 13, 669 [1959].

3 M. F. Rajewsky, W. Dauber, and H. Frankenberg, Science [Washington] 152, 83 [1966].

4 C. F. Hollander and P. Bentvelzen, J. nat. Cancer Inst. 41, 1303 [1968].

5 D. Schmähl, R. Preussmann u. H. Hamperl, Naturwissenschaften 47,89 [1960].

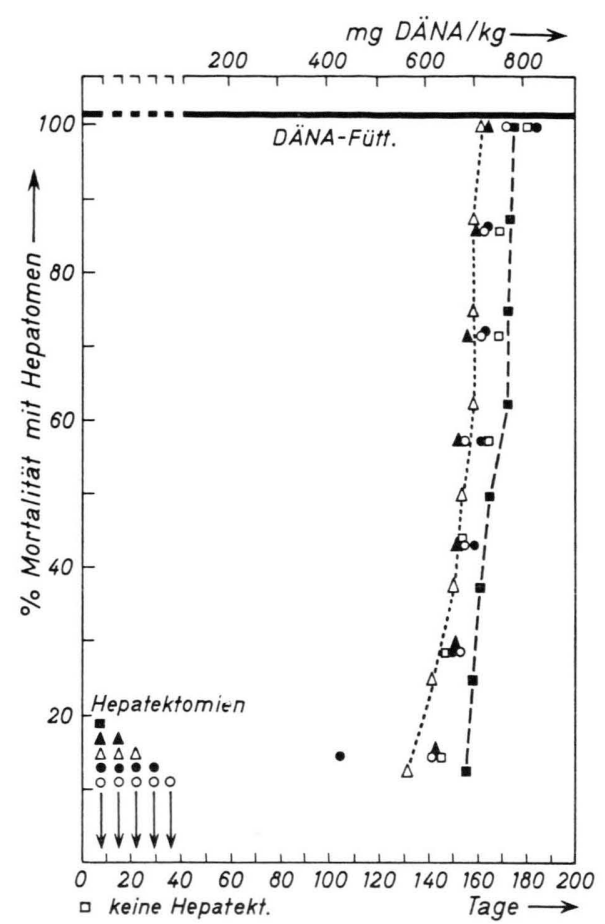

Abb. 1. Mortalität Diäthylnitrosamin-gefütterter Ratten nach partieller Hepatektomie.

Die Überlebenszeiten der Versuchstiere (Abb. 1) ließen keinen signifikanten Unterschied zwischen den einzelnen Gruppen erkennen. Lediglich zwischen den 1-mal und 3-mal operierten Tieren ergibt sich im t-Test eine schwach signifikante Differenz $(p=0,05)$.

Vor der Entstehung von carcinogen-induzierten Glucose-6-Phosphatase-Ausfallsbezirken in der Leber läßt sich durch eine 1- bis 5-mal wiederholte Resektion von Lebergewebe und die damit in wechselnder Stärke verursachte Stimulierung der Zellproliferation keine Beschleunigung der Tumorentstehung und keine deutliche Abkürzung der Überlebenszeit der Tiere nachweisen. Gleichartige Versuche nach Entstehen der Enzymausfallsbezirke sind im Gange.

Mit Unterstützung der Deutschen Forschungsgemeinschaft.

6 W. Gössner u. H. Friedrich-Freksa, Z. Naturforsch. 19 b, 862 [1964].

7 A. Schauer u. E. Kunze, Z. Krebsforsch. 70, 252 [1968].

${ }^{8}$ H. Friedrich-Freksa and M. Hoffmann, Nature [London] 223, 1162 [1969].

${ }^{9}$ H. Friedrich-Freksa, W. Gössner u. P. Börner, Z. Krebsforsch. 72, 226 [1969].

${ }^{10} \mathrm{H}$. Rabes and H. Brändle, Cancer Res. 29, 817 [1969].

${ }^{11}$ H. Rabes, P. Scholze u. R. Hartenstein, Verh. dtsch. Ges. Pathol. 57 [1970], im Druck. 